\title{
The role of self- and other-animal-produced odors in rats' preferences for novelty in an exploration box
}

\author{
ROBERT N. HUGHES \\ University of Canterbury, Christchurch, New Zealand
}

\begin{abstract}
Following confinement to one half of an exploration box, rats were faced with both halves (novel and familiar) after each half had been washed or not. When both halves had been washed, thereby decreasing the likely influence of odors arising from the earlier presence of the subject or previous occupants, neither was preferred. When only the novel half had been washed, thereby ensuring that odors were available in the familiar half alone, familiarity was preferred. However, the novel half was preferred when it contained odor cues originating from earlier occupants, and the familiar half contained no cues or cues from occupancy by the subject either alone or in combination with those from previously tested animals. The greatest preference for novelty occurred when the familiar half contained self-produced and the novel half contained other-animal-produced odors. Apparent effects of the washing procedures on rearing and locomotion were accounted for by behavioral changes that enabled the chosen half to be occupied. Since in a second experiment differences in brightness discriminability of the two halves of the apparatus did not affect novelty preferences, it was concluded that choices were made primarily on the basis of rat-produced olfactory information. When the exploration box technique described here is applied, this conclusion should be taken into consideration as one attempts to interpret the effects on novelty choices of pharmacological and surgical procedures likely to interfere with central olfactory mechanisms.
\end{abstract}

For over 40 years, investigations of the nature and causes of intrinsically motivated exploratory behavior have featured the laboratory rat more than any other species. In the study of rat exploration, the importance of collative qualities of environmental stimulation, such as novelty and complexity (Berlyne, 1960), is widely acknowledged. However, as has been pointed out by Corey (1978) and Renner (1990), many investigators continue inappropriately to employ procedures involving gross activity of indeterminate motivational origins. Such "forced exploration" (Welker, 1957) in open fields and the like cannot be undeniably related to the operation of an intrinsic exploratory tendency. But if the rat is allowed to choose freely between two or more environments differing in novelty, its preferences can indicate the extent to which its responses are directly controlled by novel stimuli in a manner appropriate for exploratory-motivated behavior.

Over a number of years, the present author has developed and utilized a technique for simultaneously recording both preferences for novelty and several forms of motor activity in the rat. Although it was originally devised to study effects on exploratory behavior of such variables as gender (Hughes, 1968a), age (Hughes, 1968b), food deprivation (Hughes \& Swanberg, 1970), and early experience (Hughes, 1971), more recently the technique has

Requests for reprints should be sent to R. N. Hughes, Department of Psychology, University of Canterbury, Christchurch 1, New Zealand. been applied to assessments of pharmacological influences (see, e.g., Horsburgh \& Hughes, 1981; Hughes, 1972; Hughes, Blampied, \& Stewart, 1975; Hughes \& Greig, 1976; Hughes \& Pither, 1987). It essentially involves a combination of spontaneous alternation (regarded as an example of exploratory behavior, Barnett, 1963) and open-field testing. By means of a behavior sampling procedure, estimates are made of the relative time spent in the familiar and mirror-image novel halves of an exploration box, along with times engaged in rearing, walking, grooming, and other specified activities. Ambulation or total distance traveled is also measured by counting the number of quadrants entered. Preferences for the novel versus familiar half of the apparatus are likely to represent exploratory-motivated choices that might occur in more natural environments where animals venture out from and retreat to familiar regions when investigating novel stimuli (Hughes, 1968a). Since novelty preferences in this situation are relatively uncontaminated by activity changes (Misslin \& Ropartz, 1981a; Robbins, 1977) and represent true novelty-approach behavior (Corey, 1978), the technique has proved useful for authors other than the writer in the estimation of effects on exploratory tendencies of manipulations that can interfere with motor activity, such as brain lesions and drug administration (see, e.g., Kirkby, 1978; Misslin, Haberkorn, \& Ropartz, 1981; Misslin, Ropartz, \& Jung, 1984; Velley, Mormede, \& Kempf, 1988).

When rats are confronted with novel and familiar halves of an exploration box or indeed when they are tested with 
most "free" exploration techniques (Welker, 1957), it is not known which particular sensory modalities are involved in the rats' choices of novelty. An important exception is spontaneous alternation, for which it has been established that intramaze novelty choices are based mainly on olfactory stimuli arising from odor trails laid down during previous trials (Douglas, 1966). Because preferences for novelty shown by mice in an exploration box are suppressed by bulbectomy and olfactory mucosa lesions (Misslin \& Ropartz, 1981b), it is likely that choices made by rats in the same and other free exploration settings would also rely heavily on olfactory cues. But the importance of visual stimuli should not be ignored, since rat exploration can be influenced by brightness changes and other visual phenomena. The present study was therefore designed to assess the importance for novelty preferences of (1) odors arising from the earlier presence of the test rat or other animals, and (2) visual cues in the form of a brightness difference between two halves of an exploration box.

\section{EXPERIMENT 1}

In addition to defecating and urinating, a rat confined to an exploration box utilized by the present author and others frequently walks and rears with its ventral surface rubbing against the floor and walls of the apparatus. Consequently, there is ample opportunity for leaving odors to be acted on later either by the rat concerned or, if the apparatus is left unwashed between subjects, by later rats. In Experiment 1, effects on behavior in an exploration box of self-produced odor cues (self) and those provided by the earlier presence of other rats (others) were investigated. To avoid replacing these cues with olfactory stimuli from chemical deodorizers or disinfectants, where need be, their strength was attenuated by appropriate washing with warm water alone.

\section{Method}

Subjects. The subjects were 60 male and 60 female albino Wistar rats approximately 130 days old. They were housed in groups of 3 or 4 same-sexed animals in 12:12-h reversed lighting at an ambient temperature of $21^{\circ}-23^{\circ} \mathrm{C}$ and relative humidity of $50 \%$. Food and water were freely available.

Apparatus. The apparatus consisted of one of four exploration boxes constructed from clear Perspex. Each box comprised four $20 \times 20 \times 20 \mathrm{~cm}$ cells formed by partial walls containing $7.5 \times 20 \mathrm{~cm}$ openings and could be divided in half through the insertion of two guillotine slides into the appropriate $7.5 \times 20 \mathrm{~cm}$ gaps in an opaque Perspex wall. The floor consisted of wire mesh, and each half of the box was covered by a hinged Perspex lid. The boxes sat in metal trays within ventilated, sound-attenuated chambers evenly illuminated by $8-W$ fluorescent lighting. Observations were made through a one-way window in the front of each chamber.

Procedure. In squads of 4 , equal numbers of males and females were individually confined for $60 \mathrm{~min}$ to one half of an exploration box (i.e., familiarization), which had or had not been washed according to the procedure described below. Each rat was then placed in a small holding cage while fecal boli were removed and counted and one or both sides of the apparatus were washed following removal of the slides separating the two halves. It was then returned to the same "familiar" half it had been confined to and was continuously observed for $10 \mathrm{~min}$. Every $5 \mathrm{sec}$, it was noted whether or not the rat was in the previously inaccessible "novel" half and whether or not it was walking, rearing, or grooming itself. The total numbers of cells entered and fecal boli dropped were also recorded.

Washing regimes for the familiar (Fam) and novel (Nov) halves of the apparatus and subsequent lack of (NC), self-produced (S). or other-animal-produced $(\mathrm{O})$ odor cues were as follows:

Familiar. Washed both before and after (Fam:NC), before but not after (Fam:S), or neither before nor after, familiarization (Fam:S\&O).

Novel. Washed (Nov:NC) or not washed (Nov:O), before both familiarization and testing.

Washing involved thorough cleaning of the floor, walls, lids, and metal tray for the appropriate side(s) of each exploration box with a sponge and warm water.

Equal numbers of subjects were randomly assigned to the six washing conditions. Half of each group were familiarized with the left and half with the right side of an exploration box. Testing was carried out during the dark phase of the light:dark cycle on which the animals were maintained.

\section{Results}

Results for all measures except defecation during familiarization can be seen in Figure 1. All data outlined were subjected to separate 3 (washing of the familiar half) $\times 2$ (washing of the novel half) $\times 2$ (sex) analyses of variance (ANOVAs).

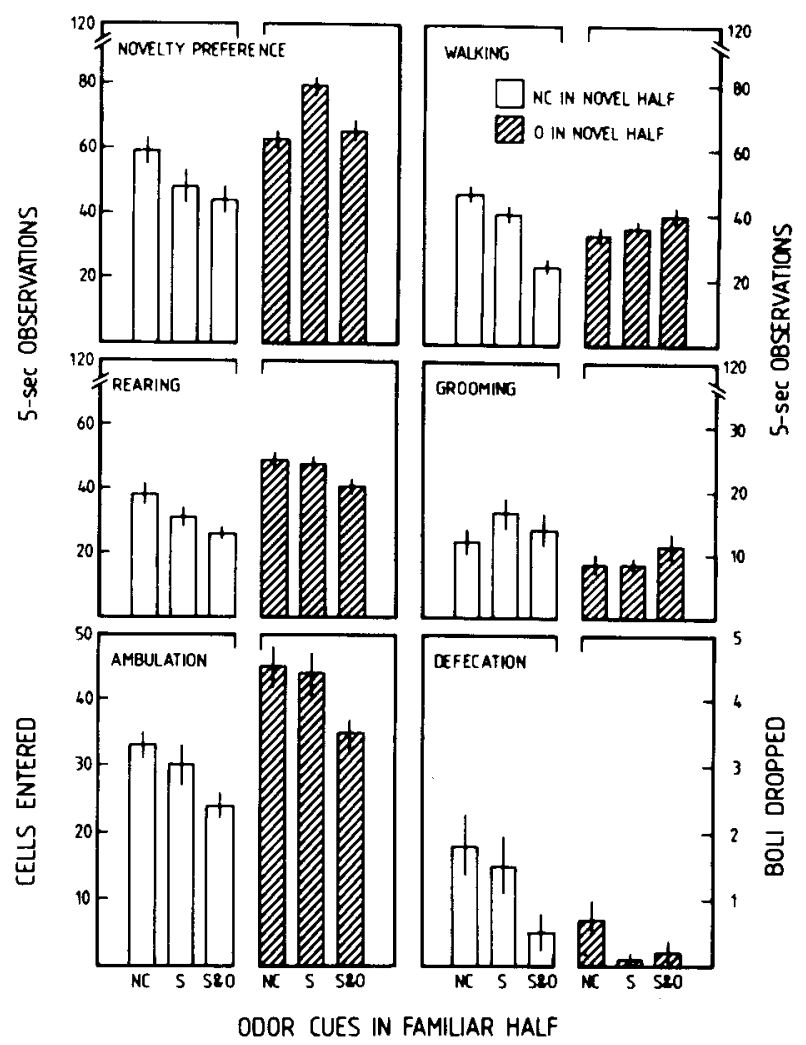

Figure 1. Mean $( \pm S E M)$ scores on all measures when either half or both halves contained attenuated odor cues (NC), self-produced cues (S), other-animal-produced cues $(O)$, or self- plus other-animalproduced cues (S\&O). 
Novelty preference. This measure was significantly affected by the washing treatment of both the familiar $[F(2,108)=3.39, p<.05]$ and the novel halves of the apparatus $[F(1,108)=41.25, p<.0001]$. In view of a significant interaction between washing of the familiar half and washing of the novel half $[F(2,108)=7.20, p<$ $.01]$, separate one-way ANOVAs were performed on preferences observed following washing of the familiar half for each type of treatment of the novel half. These revealed that, when there were no cues in the novel half (Nov:NC), the different forms of treatment of the familiar half did not influence preference scores $[F(2,57)=2.88]$. However, when the novel half contained odors of other rats (Nov:O), treatment of the familiar half significantly affected the rats' choices $[F(2,57)=13.59, p<.0001]$. According to Dunnett $t$ tests, this effect arose from significantly higher preferences shown by rats returned to a familiar half containing self-produced odors (Fam:S) as opposed to those returned to one containing either no odors (Fam:NC) or self- and other-animal-produced cues (Fam:S\&O).

Wilcoxon $T$ tests showed that rats faced with the Nov:NC and Fam:S or Fam:S\&O combination preferred to occupy the familiar rather than the novel half $(z=1.99, p=.05$; $z=2.89, p<.01)$. When both halves had been washed prior to testing (Nov:NC, Fam:NC), neither was preferred $(z=.28)$. However, of the groups allowed access to an unwashed novel half (Nov:O), two showed significant preferences and one a marginally significant preference for being in the novel side of the apparatus (Fam:NC, $z=$ $1.91, p=.056$; Fam:S, $z=3.93, p<.001$; Fam:S\&O, $z=2.16, p=.031$ ).

Walking. Walking frequencies were significantly affected by treatment of the familiar $[F(2,108)=10.38$, $p<.001]$ but not the novel half $[F(1,108)=.02]$. Because of a significant interaction between washing of the two halves $[F(2,108)=29.88, p<.0001]$, one-way ANOVAs similar to those performed for novelty preference were also carried out on walking scores. Treatment of the familiar half had a significant effect when the novel half was relatively odorless [Nov:NC, $F(2,57)=34.93$, $p<.0001]$, but not when it contained other animal cues [Nov:O, $F(2,57)=2.05]$. Dunnett $t$ tests showed the significant effect to be due to less walking by rats in the Fam:S\&O condition.

Rearing. This response was significantly affected by treatment of both halves of the apparatus [familiar half, $F(2,108)=10.05, p<.001$; novel half, $F(1,108)=$ $55.16, p<.0001]$. A significant interaction between washing of the familiar half and sex for rearing $[F(2,108)$ $=3.73, p<.03$ ] followed by one-way ANOVAs and Dunnett $t$ tests revealed that, while this measure was unaffected for females by the washing procedure $[F(2,57)=$ 1.12], males in the Fam: $S \& O$ condition $(M \pm S E M=$ $29.15 \pm 2.46)$ reared less often than those in the Fam:NC $(43.55 \pm 2.13)$ or $F a m: S$ conditions $[41.30 \pm 2.94, F(2,57)$ $=9.38, p<.001]$. Females $(37.6 \pm 2.31)$ reared more often than males $(29.15 \pm 2.45)$ only in the Fam:S\&O condition.

As can be seen in Figure 1, the significant effect on rearing of washing the novel half arose from higher frequencies of the activity when it was left unwashed (Nov:O).

Grooming. Grooming was unaffected by either washing of the familiar half $[F(2,108)=.84]$ or sex $[F(1,108)$ $=.02]$. However, the rats groomed more often in the Nov:NC than in the Nov:O condition $[F(1,108)=7.80$, $p<.01]$.

Ambulation. The total distance traveled was significantly affected by treatment of both the familiar $[F(2,108)=8.66, p<.001]$ and the novel halves of the apparatus $[F(1,108)=38.95, p<.0001]$. As shown by Dunnett $t$ tests, rats that were returned to a familiar half left unwashed both before and after familiarization (Fam:S\&O) ambulated less than those in the other two groups (see Figure 1). They also ambulated more in the Nov:O than in the Nov: $N$ condition.

Defecation. $M \pm S E M$ numbers of fecal boli dropped during familiarization by rats confined to a washed or unwashed familiar half were $4.05 \pm .38$ and $3.30 \pm .43$, respectively. This difference was not significant $[t(118)=$ 1.24]. During tests, defecation was unaffected by the washing procedures. However, during familiarization, males (4.92 \pm .42$)$ defecated more often than did females $[2.59 \pm .33, F(1,108)=19.31, p<.0001]$.

\section{Discussion}

It is clear from the novelty-preference results of this experiment that, in the absence of obviously discriminable visual cues, the rats' choices of the novel or the familiar half of an exploration box were based on odor cues arising from the earlier presence of test or other animals. When the rats were faced with attenuated cues in both halves, no preference was shown for either alternative. But if cues from a test rat, either alone or combined with those from previous occupants, were present in the familiar half while it was allowed access to a washed and comparatively odorless novel half, the rat preferred to remain where rat-generated olfactory stimuli were still available. However, when cues from animals tested earlier were present in the novel half, this was preferred regardless of whether or not self- or other-animal-produced cues were present in the familiar half. The highest preference for novelty occurred when the familiar half contained mainly self-produced cues. Although a washed familiar half and an unwashed novel half provided the greatest olfactory contrast between the two alternatives, it could be construed that self-produced familiar versus other-animalproduced novel cues represented the greatest novelty difference. This is because, when rats are faced with both an odiferous and a comparatively odorless alternative, their preference for the former could be entirely unrelated to its novelty value and may merely reflect the rat's natural attraction to sources of olfactory stimulation. Such an 
interpretation would account for the familiarity preferences observed in the present experiment, but it would not readily explain preferences for the novel side when odor cues were available in both halves of the apparatus. It therefore seems likely that any decisions concerning novelty were based mainly on the availability of ratgenerated olfactory stimuli.

While more difficult to account for by means of an exploratory motive alone, the measures of motor activity were also affected, to varying degrees, by manipulations of the rats' olfactory environment. Only when faced with a relatively odorless novel half did the rats that were returned to a familiar half containing both self- and otheranimal-produced cues show decreased frequencies of walking. However, irrespective of the nature of the familiar half, overall, rearing was greater when the novel half contained cues from rats tested earlier. Presumably this was due to the presence of cues on the walls and lids that encouraged the behavior. However, this would not account for why males (but not females) reared less when the familiar half contained self- and other-animalproduced cues regardless of what characterized the novel half. Since grooming is viewed by some authors as an example of conflict-induced displacement (Barnett, 1963), the higher frequency of the behavior shown by rats given access to a relatively odorless novel half, compared with one containing other-animal-produced cues, might suggest indecision in the face of insufficient olfactory information. This is supported by the lack of preference shown by these subjects for either half of the apparatus or by their tendencies to occupy the familiar half, where cues were present.

As the ambulation results show, the rats that were returned to a familiar half containing both self- and otheranimal-produced cues traveled the shortest distances. This also typified animals given access to a comparatively odorless novel half. Although it is possible that these outcomes reflected some locomotor-inhibiting influence of familiar odors on the one hand and insufficient olfactory information on the other, as for the related walking measure, they cannot be adequately accounted for at the present time.

In view of the general acceptance of defecation as an index of emotionality (Archer, 1973; Walsh \& Cummins, 1976) and the lack of effects of washing on fecal boli dropped during either familiarization or testing, it is unlikely that any of the behavioral results described were due to changes in emotional reactivity. Instead, it is possible that effects of the experimental treatments on walking, rearing, and ambulation were largely determined by the extent to which these activities enabled the rats to make their olfactory choices, since each measure was significantly correlated with novelty preference [walking, $r(118)$ $=.43, p<.01$; rearing, $r(118)=.50, p<.01$; ambulation, $r(118)=.54, p<.01]$. Whatever the case, it is clear that, as with spontaneous alternation (Douglas, 1966), olfactory cues play an important part in location choices made by rats that are provided with two environments differing in novelty.

\section{EXPERIMENT 2}

Even though rats may utilize mainly odors for selecting the more novel of two environments, there is ample evidence of their ability to make such choices on the basis of visual information alone. For example, when rats are allowed to see into the goal arms of a T-maze while being prevented from entering them, and are then given free access to each arm, they select the arm that has been changed in brightness (Dember, 1956; Dember \& Millbrook, 1956; Kivy, Earl, \& Walker, 1956) or pattern (Earl, Franken, \& May, 1967; May, 1968). Rats also show more exploratory behavior in visually complex environments than they do in less complex environments (Dember, Earl, \& Paradise, 1957; Lester, 1967; Woods \& Davidson, 1964). Therefore, in Experiment 2, the role of a brightness difference between walls of the two halves of an exploration box was investigated in combination with odor cues provided by the two washing procedures that in Experiment 1 produced either the highest preference for novelty (Fam:S, Nov:O) or no preference for either half (Fam:NC, Nov:NC).

\section{Method}

Subjects and Apparatus. The subjects were an additional 24 male and 24 female Wistar albino rats approximately 125 days old. They were housed in the same conditions and tested in the same apparatus as were the rats in Experiment 1. In addition, to provide two conditions of visual discriminability, $40 \times 20 \mathrm{~cm}$ cards were attached to the outside of the transparent back and sidewalls of each box. These were either medium gray all over, or black for one half of a box and white for the other.

Procedure. The general procedure was the same as it was for Experiment 1. Half of each sex were tested with the novel side of the apparatus left unwashed (Nov:O) and the familiar side washed before but not after familiarization (Fam:S). For the other half, both sides were washed before and after familiarization (Fam:NC, Nov:NC). Gray cards (low discriminability) were attached to both sides during familiarization and testing for half the subjects, and a black card was attached to one and a white to the other for the remainder (high discriminability).

\section{Results}

The results for all measures except defecation during familiarization are outlined in Figure 2.

Both the washing procedure and sex significantly affected preferences for occupying the novel half of the apparatus [washing, $F(1,40)=21.20, p<.0001$; sex, $F(1,40)=4.70, p<.04]$. These results were due to higher preferences shown by rats confronted with selfproduced odors in the familiar (Fam:S) and other-animalproduced odors in the novel half (Nov:O) than by rats for which both halves had been washed before testing (Fam:NC, Nov:NC). Females $(M \pm S E M,=71.21$ \pm 2.83 ) showed higher preferences than did males $(63.21 \pm 3.25)$. However, effects of visual discriminabil- 


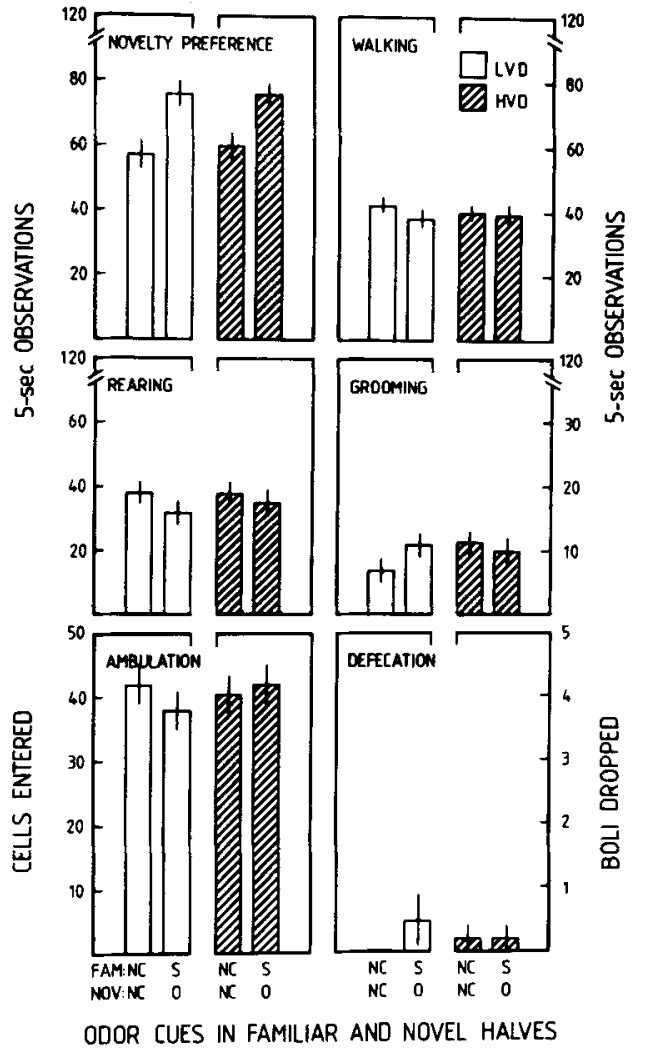

Figure 2. Mean $( \pm S E M)$ scores on all measures in low and high visual discriminability conditions (LVD, HVD) when neither half contained odor cues (FAM:NC, NOV:NC) or when the familiar half contained self-produced and the novel half contained other-animalproduced cues (FAM:S, NOV:O).

ity of the two halves were not significant $[F(1,40)=.22]$. Although for each level of visual discriminability, rats tested with odor cues available in both halves (Fam:S, Nov:O) showed significant preferences for novelty (low, $z=2.49, p<.02$; high, $z=2.94, p<.01$ ), neither half was preferred when odor cues were minimal (i.e., Fam:NC, Nov:NC: low, $z=.90$; high, $z=0$ ). No other measures were affected by the experimental treatments.

There were significant sex differences for walking $[F(1,40)=6.80, p<.02]$, rearing $[F(1,40)=11.43$, $p<.01$ ], and defecation [during familiarization, $t(46)=$ $2.06, p<.05]$. These were due to higher walking $(M \pm S E M=42.21 \pm 1.46)$ and defecation $(2.5 \pm .51)$ and lower rearing (31.21 \pm 1.88$)$ for males than for females (walking $=37.54 \pm 1.01$, defecation $=1.04 \pm .48$, rearing $=40.58 \pm 2.04$ ). Although no other sex main effects were significant, significant interactions occurred between sex and washing on the one hand $[F(1,40)=5.04, p<$ $.04]$, and between sex and visual discriminability on the other $[F(1,40)=5.97, p<.02]$. Dunnett $t$ tests revealed significantly more ambulation for males $(43.67 \pm 2.63)$ than for females $(35.42 \pm 2.29)$ only when they were faced with the Fam:S and Nov:O combination, and when one half was black and the other white (males $=45.33 \pm 2.03$, females $=36.58 \pm 2.82$ ). Males (but not females) also ambulated more when the halves were black and white $(45.33 \pm 2.03)$ than when both were gray $(38.08 \pm 2.24)$.

\section{Discussion}

As occurred in Experiment 1, preferences for occupying the novel half were evident only if odor cues were present in each half of the apparatus. In this case, familiar cues were primarily self-produced, whereas novel cues arose entirely from the earlier presence of other animals. However, visual cues appeared to be of little importance in determining the rats' choices, since differences in discriminability between the two halves had no effect on which one they preferred. Although it is possible that the brightness contrast between the two halves characterizing high discriminability was insufficient to distinguish it from the low discriminability condition, this seems unlikely in view of the difference in ambulation between the two conditions shown by males. Besides, it is well established that exploring rats have no difficulty in distinguishing between areas enclosed by black or white walls (see, e.g., Dember, 1956; Kivy et al., 1956; Williams \& Kuchta, 1957).

Although the higher preference for novelty shown by females in Experiment 2 (but not Experiment 1) has not generally characterized research utilizing the specific technique under consideration, a similar sex difference has been described in other novelty-choice settings (see, e.g., Richards \& Leslie, 1962; Russell, 1977). However, it has also recently been shown that, with a shorter familiarization period than that adopted here, females exhibit higher preferences than do males in an exploration box, because of their more rapid habituation to the familiar half (Hughes, in press).

Contrary to what was observed in Experiment 1, the washing procedure had no significant effects on measures of activity. This was probably because, in the statistical analyses, characteristics of the familiar and the novel halves were not treated as separate factors. Consequently, effects observed earlier could have been obscured by the particular combination of washing procedures adopted. For example, whereas in Experiment 1 a familiar side containing self- and other-animal-produced odors (Fam:S\&O) was accompanied by less walking when the novel side was comparatively odorless (Nov:NC), washing of the familiar half had no effect when the novel half contained other-animal-produced odors (Nov:O). Only that part of the latter treatment of the two halves which had an effect on walking (i.e., Fam:S and Nov:O) was included in Experiment 2. Although females in Experiment 1 reared more often than males only when they were returned to a familiar half containing self- and otheranimal-produced odors (Fam:S\&O), the same sex difference occurred for rearing (as well as for walking) in Experiment 2, regardless of how the familiar half had been treated. Again females defecated less often during familiarization, and, contrary to what had occurred previously, they walked less often than males did. Although similar 
sex differences in rearing and defecation have been described by other workers, females usually engage in more rather than less locomotor activity than males do (Archer, 1975).

\section{GENERAL DISCUSSION}

The results of these two experiments clearly indicate that preferences shown by normal, intact rats for occupying the more novel half of the type of exploration box utilized by the present author and others are mainly determined by the nature of rat-produced odor cues. Since in most previous research it has been usual to leave both halves of the apparatus unwashed at all times, it is interesting that, although this procedure still gave rise to significant preferences for novelty, they were lower than when the familiar half was washed before but not after familiarization. To avoid floor effects, it would be advisable to adopt the latter procedure if a particular experimental manipulation was expected to suppress responsiveness to novelty.

The significance of self-produced odors in determining later location choices demonstrated here has also been shown for spontaneous alternation behavior (Douglas, 1966). However, the present results indicate that odors arising from the earlier presence of conspecifics can be equally significant.

The importance of olfaction for rats in an exploration box supports similar conclusions for mice following bulbectomy and olfactory mucosa lesions (Misslin \& Ropartz, 1981b). These authors concluded that their operated animals appeared to be unable to discriminate between novel and familiar stimuli. A similar conclusion was also reached for mice by Maruniak, Darney, and Bronson (1975) and Schoots, Crusio, and van Abeelen (1978)although they did make their observations in different experimental settings.

In view of the often suspect validity of activity measures as indices of an intrinsic exploratory tendency (Corey, 1978; Hughes, 1982), it would be unwise to conclude that the changes in rearing and locomotor activity associated with the various washing procedures in Experiment 1 directly reflected effects of odor cues on the rats' motivation to explore. Instead, it is more likely that levels of these responses were determined by the extent to which they were necessary for enabling occupancy of the chosen half of the apparatus. The relative independence of the activity and choice measures is supported by the lack of any effects of the washing procedure on rearing, walking, and ambulation in Experiment 2 accompanied by significant changes in preferences for novelty. Although some investigators have paid attention to proportions of vertical and horizontal activities occurring in novel and familiar environments (e.g., Bardo, Neisewander, \& Pierce, 1989; Pierce, Crawford, Nonneman, Mattingly, \& Bardo, 1990), the present author has failed to observe any advantage of employing this distinction instead of totals for each half of an exploration box.
Although inconsistent with results of response-to-change experiments (Dember, 1956; Dember \& Millbrook, 1956; Kivy et al., 1956), the failure of a brightness difference between the two halves of the apparatus to have any effects on preferences for novelty in Experiment 2 is in line with the inconclusive role of intramaze visual cues for the related phenomenon of spontaneous alternation (Richman, Dember, \& Kim, 1987). Even though the difference in ambulation between the visual conditions indicated that, for males at least, they were discriminable, it would be useful to investigate the role of pattern as well as brightness differences.

From the results of the present study, it can be concluded that the exploration box procedure for the investigation of intrinsic exploratory tendencies in rats relies primarily on the availability of self- and other-animalproduced odors. Visual cues in the form of brightness differences seem to be of little importance. This information should therefore be taken into consideration in any attempts to interpret the results of experiments involving surgical or pharmacological manipulations of central mechanisms related to olfaction as well as behavior, as in the cases of lesion or anticholinergic drug effects on some limbic structures (Hughes, 1982).

\section{REFERENCES}

ARCher, J. (1973). Tests for emotionality in rats and mice: A review. Animal Behaviour, 21, 205-235.

ARCHER, J. (1975). Rodent sex differences in emotional and related behavior. Behavioral Biology, 14, 451-479.

Bardo, M. T., Neisewander, J. L., PierCe, R. C. (1989). Noveltyinduced place preference in rats: Effects of opiate and dopaminergic drugs. Pharmacology, Biochemistry \& Behavior, 32, 683-689.

BARNeTT, S. A. (1963). A study in behavior. London: Methuen.

Berlyne, D. E. (1960). Conflict, arousal, and curiosity. New York: McGraw-Hill.

CorEY, D. T. (1978). The determinants of exploration and neophobia. Neuroscience \& Biobehavioral Reviews, 2, 235-253.

DEMBER, W. N. (1956). Response by the rat to environmental change. Journal of Comparative \& Physiological Psychology, 49, 93-95.

Dember, W. N., Earl, R. W., \& Paradise, N. (1957). Response by rats to differential stimulus complexity. Joumal of Comparative \& Physiological Psychology, 50, 514-518.

Dember, W. N., Millbrook, B. A. (1956). Free-choice by the rat of the greater of two brightness changes. Psychological Reports, 2 , 465-467.

Douglas, R. J. (1966). Cues for spontaneous alternation. Journal of Comparative \& Physiological Psychology, 62, 171-183.

EArL, R. W., Franken, R. E., \& MAY, R. B. (1967). Choice as a function of stimulus change. Perceptual \& Motor Skills, 24, 183-189.

HoRSBURGH, R. J., \& HugHES, R. N. (1981). Modification of novelty preferences in rats by current and prior treatment with scopolamine and methylscopolamine. Psychopharmacology, 73, 388-390.

HuGHes, R. N. (1968a). Behaviour of male and female rats with free choice of two environments differing in novelty. Animal Behaviour, 16, 92-96.

HuGHES, R. N. (1968b). Effects of age on novelty reactions and exploration in rats. Quarterly Journal of Experimental Psychology, 20, 189-192.

HuGHES, R. N. (1971). Reactions to novelty following differential postweaning rearing and handling. Perceptual \& Motor Skills, 32, 883-886.

Hughes, R. N. (1972). Chlordiazepoxide modified exploration in rats. Psychopharmacologia, 24, 462-469.

Hughes, R. N. (1982). A review of atropinic drug effects on explor- 
atory choice behavior in laboratory rodents. Behavioral \& Neural Biology, 34, 5-41.

HuGHEs, R. N. (in press). Sex-dependent habituation to novelty in rats. Current Psychology: Research \& Reviews.

Hughes, R. N., Blampied, N. M., \& Stewart, W. J. (1975). Scopolamine induced changes in activity and reactions to novelty. Pharmacology, Biochemistry \& Behavior, 3, 731-734.

Hughes, R. N., Greig, A. M. (1976). Effects of caffeine, methamphetamine and methylphenidate on reactions to novelty and activity in rats. Neuropharmacology, 15, 673-676.

Hughes, R. N., \& Pither, J. M. (1987). Chronic imipramine effects on exploratory behavior in rats. Pharmacology, Biochemistry \& Behavior, 27, 359-362.

Hughes, R. N., \& Swanberg, K. M. (1970). Effects of food deprivation in exploration in deprivationally naive rats. Australian Journal of Psychology, 22, 79-84.

KIRKBY, R. J. (1978). Exploratory behavior in rats following bilateral lesions of the anterodorsal caudate nuclei. Perceptual \& Motor Skills, 47, 623-628.

KIVy, P. N., EArL, R. W., \& WALker, E. L. (1956). Stimulus context and satiation. Joumal of Comparative \& Physiological Psychology, 49, 90-92.

LESTER, D. (1967). Exploratory response of rats to stimuli of differing complexity. Perceptual \& Motor Skills, 24, 1333-1334.

MARUniaK, J. A., DARney, K. J., JR., \& Bronson, F. H. (1975). Olfactory perception of the nonsocial environment by male house mice. Behavioral Biology, 14, 237-240.

MAY, R. B. (1968). Pretest exposure, changes in pattern complexity, and choice. Joumal of Comparative \& Physiological Psychology, 66, 139-143.

Misslin, R., Haberkorn, E., \& Ropartz, P. (1981). Responses to novelty and changes in behavior across a 3 -week postoperative period in hippocampal-lesioned mice. Physiology \& Behavior, 27, 413-418.

Misslin, R., \& RopARTz, P. (1981a). Effects of methamphetamine on novelty-seeking by mice. Psychopharmacology, 75, 39-43.

Missuin, R., \& RoparTZ, P. (1981b). Olfactory regulation of responsiveness to novelty in mice. Behavioral \& Neural Biology, 33, 230-236.

MissLin, R., RoparTz, P., \& JUNG, L. (1984). Impairment of responses to novelty and its antagonism by neuroleptics in mice. Psychopharmacology, 82, 113-117.
Pierce, R. C., Crawford, C. A., Nonneman, A. J., Mattingly, B. A., BARDO, M. T. (1990). Effect of forebrain dopamine depletion on novelty-induced place preference behavior in rats. Pharmacology, Biochemistry \& Behavior, 36, 321-325.

RENNER, M. J. (1990). Neglected aspects of exploratory and investigatory behavior. Psychobiology, 18, 16-22.

Richards, W. J., \& LesLie, G. R. (1962). Food and water deprivation as influences on exploration. Journal of Comparative \& Physiological Psychology, 55, 834-837.

Richman, C. L., Dember, W. N., \& KIM, P. (1987). Spontaneous alternation behavior in animals: A review. Current Psychological Research \& Reviews, 5, 358-391.

Robiss, T. W. (1977). A critique of the methods available for the measurement of spontaneous motor activity. In L. L. Iversen, S. D. Iversen, \& S. H. Snyder (Eds.), Handbook of psychopharmacology: Vol. 7. Principles of behavioral pharmacology (pp. 37-82). New York: Plenum.

RuSSELL, P. A. (1977). Sex differences in rats' stationary exploration as a function of stimulus and environmental novelty. Animal Learning \& Behavior, 5, 297-302.

Schoots, A. F. M., Crusio, W. E., \& van Abeelen, J. H. F. (1978) Zinc-induced peripheral anosmia and exploratory behavior in two inbred mouse strains. Physiology \& Behavior, 13, 407-425.

Velley, L., Mormede, P., \& KempF, E. (1988). Neurochemical lesion of the nucleus locus coeruleus increases neophobia in a specific exploration task but does not modify endocrine response to moderate stress. Pharmacology, Biochemistry \& Behavior, 29, 1-7.

Walsh, R. N., \& Cummins, R. A. (1976). The open-field test: A critical review. Psychological Bulletin, 83, 482-504.

WeLKER, W. I. (1957). "Free" versus "forced" exploration of a novel situation by rats. Psychological Reports, 3, 95-108.

Williams, C. D., KuchTA, J. C. (1957). Exploratory behavior in two mazes with dissimilar alternatives. Journal of Comparative \& Physiological Psychology, 50, 509-513.

Woods, P. J., Davidson, E. H. (1964). The behavioural effects of changes in environmental complexity. Canadian Journal of Psychology, 18, 23-27.

(Manuscript received October 15, 1990; revision accepted for publication December 19, 1990.) 